\title{
Review on the Test Method of Adhesive Failure in Solid Rocket Motor
}

\section{Chen ${ }^{1, a^{*}}$, LU Gui-e ${ }^{1,2}$,Jiang Jinyong ${ }^{1,2}$, Ge Qiang ${ }^{1,2}$, Wang Shaoguang ${ }^{1,2}$,}

\author{
Wang Bin ${ }^{1,2}$ \\ ${ }^{1}$ Ordnance Engineering College, Shijiazhuang 050003, China; \\ 2Ordnance Technical Research Institute, Shijiazhuang 050003, China \\ alichen19910108@163.com,
}

Keywords: solid rocket motor, debonding, infrared heat wave, ultrasonic, laser holographic interferometry, industry CT, $\mathrm{X}$ ray

\begin{abstract}
This paper introduced common detection methods of adhesive failure in solid rocket motor including ultrasonic method, $\mathrm{X}$ ray radiography technique, industrial $\mathrm{CT}$, infrared heat wave method and laser holographic interferometry. It also recommended the most suitable detection methods for various types of debonding.
\end{abstract}

\section{Introduction}

Solid propellant is a kind of high-energy material which is widely used in aerospace and military area. In order to make sure the combustion of propellant develops in booked way and prevent the rocket motor from high temperature nozzle jet, propellant is usually covered with one or more coatings (generally consist of insulation layer and liner)[1,2]. In the processing of cooling solidification, long-distance transportation, long-time storage and service, the interface between propellant, coating and steel shell may occur adhesive failure in some area $[3,4]$. One third of the failure of solid rocket motor test abroad is caused by interface adhesive failure and the major failure mode is debonding between propellant and liner [5]. Therefore, it is important to detect the adhesive failure in solid rocket motor validly.

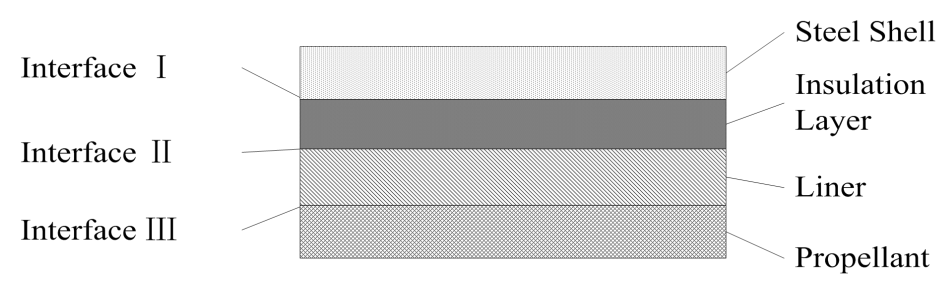

Fig.1 Figure of adhesive structure of propellant

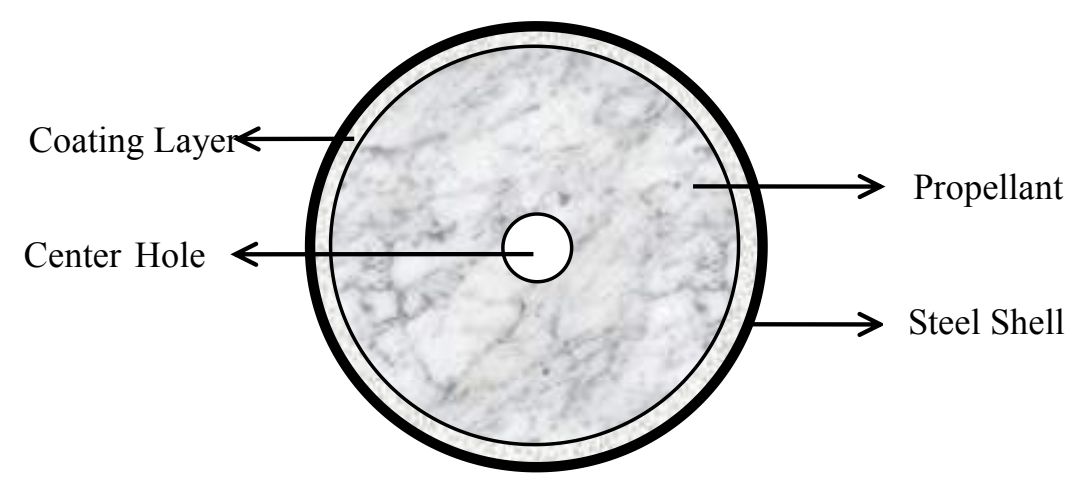

Fig.2 Figure of adhesive structure of Solid Rocket Motor 
The common test methods of adhesive failure in rocket motor include ultrasonic method, $\mathrm{X}$ ray radiography technique, industrial $\mathrm{CT}$, infrared heat wave method, laser holographic interferometry, microwave method, electronic speckle pattern interferometry and so on based on different testing principle. These methods will be introduced in detail in this paper.

\section{Infrared Heat Wave Method}

\subsection{Test Principle}

In recent ten years, infrared heat wave method developed reliably and widely used in many areas. In the process of infrared heat wave test, external heat producer heats the surface of test specimen while infrared thermal imager records the temperature field of the surface of test specimen. The real-time infrared thermal image will be shown on computer [6-8]. If adhesive failure exists, brightness appeared in thermal image for the lower conductivity of air compared with rubber.

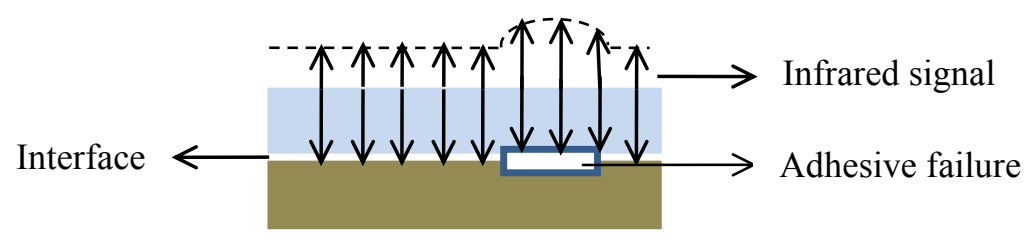

Fig.3 The principle of infrared heat wave test

\subsection{Research Situation}

Jiang Shufang[6] studied on test specimen of solid rocket motor with artificial simulated debond by infrared heat wave method. The test specimen simulated adhesive failure between rocket motor shell and heat insulating layer(adhesive failure of interface I ). It also simulated debond between heat insulating layer and inner insulation layer(adhesive failure of interface II ). Test showed that infrared heat wave method can detect adhesive failure of interface I effectively. But adhesive failure of interface II cannot be detected.

Fan Dandan[9] did research into thermal excitation method of infrared thermal wave by numerical simulation. Finite element analysis software COMSOL was used to simulate situation excited by different thermal excitation. The study indicated that pulsed excitation has the best detectability and continuous heat excitation has the worst detectability. Under the condition of lock-in thermography, adhesive failure can be detected effectively while it needed larger data collection and bigger data process load.

Guo Xingwang[10] performed numerical modeling about infrared heat wave method detecting cladding debond based on finite element analysis. The test researched into the relationships between thermal excitation method, structure parameters of test specimen and detectability and put forward that detecting condition can be optimized by computer simulating.

Jin Guofeng[11]detected composite materials interface kissing type damages by ultrasonic infrared thermal wave method. It turned out that ultrasonic infrared thermal wave method had a good detectability to interface kissing damage. But it has no detection result for debonding damage.

Wu Cuiqin[12] estimated debond defects of glass fiber composite materials applied in the SRM by infrared thermography nondestructive testing method. The results show that the infrared thermography is a fast and effective inspection method for detecting debond with the diameter over $10 \mathrm{~mm}$ and depth within $5 \mathrm{~mm}$. 


\section{Ultrasonic Method}

\subsection{Test Principle}

Reflection, refraction, diffraction and wave-type conversion may occur when ultrasonic meet the interface of two medium with different acoustic impedance. Ultrasonic method judges the quality of inside test specimen through time domain analysis or frequency domain analysis $[13,14]$. Common ultrasonic methods include echo-pulse method, Lamb wave method, slant longitudinal ultrasonic wave, acousto-ultrasonic method and so on [15]. Up to now, multiple results have been achieved by researchers of North University of China.

\subsection{Research Situation}

Professor Wang Zhaoba [16] detected the quality and thickness of liner from outside rocket steel shell by Lamb wave method in 2001. The study showed that Lamb wave method can either detect the thickness of singer or several coating layers or the adhesive failure between different layers.

Chen Youxing [17] researched on the signal processing of Lamb wave and Longitudinal wave when detecting multi-layered adhesive interface. The advantages and disadvantages of Lamb wave and Longitudinal wave method were compared. A proposal was put forward that we'd better combine Lamb wave and Longitudinal wave when detecting test specimen.

Yuan Hua [15] used Longitudinal Wave method, the Lamb Wave method and the Transverse Wave method to test $2 \mathrm{~mm}, 5 \mathrm{~mm}, 7 \mathrm{~mm}$ thick shell component of the multi-layered adhesive interface. FIR filter technology and Hilbert transform technology were applied to extract various layered characteristic signal. The conclusions were drew that Longitudinal Wave method can only examine the first interface debonding of multi-shell thickness effectively and Lamb Wave method can only examine $2 \mathrm{~mm}$ thick shell of the three interface debonding and Transverse Wave method can effectively detect different thickness of the shell interface bonding state by adjusting the distance and angle of the probe and using features of the signal extraction method.

Huang Pei [18] applied the phased array ultrasonic technology to study the issue of multi-layered interface debonding. Through the establishment of related model of phased array ultrasonic technology, the paper simulatedly examined the sound field properties of the multi-media phased array transducer and the process of detecting multi-layered debonding components.

Zhang Peng [19] studied on the extractive method of debonded signal through cross correlation technology and EMD method and exploring debonding-imaging processing technology through BEMD method. A foundation for the precise location and the area measuring of debonding area was provided in the text.

Ai Chun-an[20] established an acousto-ultrasonic experimental system to detect the interface defects between case and insulation of SRM. In the experiment, effective detection of the defects can be realized as well as qualitative assessment of circular port size and localization of debonding defect. The result showed that the acousto-ultrasonic method can effectively detect the interface defects.

$\mathrm{Lu}$ Minghui[21] improved the penetrating capability of ultrasonic wave in viscoelastic medium by using low frequency transducers. The experimental results showed that ultrasonic feature scan imaging system can successfully be used for detecting defects of grain by means of amplitude imaging and depth imaging. 


\section{X-ray Method and Industrial CT Method}

\subsection{Test Principle}

X-ray decayed when penetrating the test specimen for different penetration ranges and materials. The quality of test specimen was got by analyzing the ray intensity of different part of specimen. X-ray diffraction data of different incident angles can be obtained by changing the relative location or direction of specimen and radiation source. After image reconstructing by computer, the three-dimentional CT image can be achieved[22].

\subsection{Research Situation}

Zheng Wei[23] put forward that the adhesive failure can be detected by X-ray method combined with ICT method. The result was got that X-ray method had an advantage of detecting sensitivity compared with ICT method. But ICT method can show the complete morphology of defect while X-ray method cannot do it.

Ai Chun-an[24] put forward that filter back projection algorithm can be used to reconstruct image of the region we interested based on the information of X-ray film. The simulation result showed that this method was effective for tangential defect especially the debonding in Solid Rocket Motor on the occasion of limited-angle and tangential detection.

Liu Fugang[25] analysed the process of detecting solid rocket motor through high energy X-ray method, and came up with the optimization of the way taking photos, the choice of film and intensifying screen. It also pointed out that scattered rays should be controlled and cumulative radiation should be estimate and record in consideration of influence that excessive radiation may have on propellant.

Zhang Lihan[26] detected the interface debonding between the propellant surface and its coating by X-ray tangent detection. Through theoretical analysis and trial and error, the detecting condition was optimized. It proved that X-ray tangent detection was feasible and the testing result was accurate and reliable.

\section{Laser Holographic Interferometry}

\subsection{Test Principle}

In laser holographic interferometry test, specimen was put into vacuum chamber with laser irradiation. The debonding part between cladding and propellant would get swell slowly during vacuumizing. According to the elasticity modulus of cladding, the degree of vacuum, the thickness of cladding and so on, the area of debonding can be calculated based on finite element method[27].

\subsection{Research Situation}

Wang Dongsheng[28] studied on the holograph of debonding in thick-cladding grains in laser holographic interferometry test. The thickness of cladding and the influence of debonding area to the deformation of grains had been confirmed in test. It indicated that laser holographic interferometry can effectively detect the debonding less than $8 \mathrm{~mm}$ of grains with its cladding thicker than $4 \mathrm{~mm}$.

$\mathrm{Wu}$ Wenjun[29] analyzed the principle and course of laser shearography and gave some improvement to test bonding quality efficiently. According to the experiment, the debonding area greater than $3 \mathrm{~mm}$ could be detected by the improved system.

$\mathrm{Li}$ Huijuan[30] used shearography to inspect specimen with known debonding size. The measurement result shows that the measurement is equal to the trim size and the accuracy is less than $10 \%$. The shearography method could meet the need of detecting honeycomb structure 
products.

Zi Peng[31] designed a series algorithm including image segmentation and image feature extraction for the feature of holographic fringe image. Zi Peng also developed a test system which used for holographic detection in the $\mathrm{Vc}++$ software. The results revealed that quanitiative system is effective and can identify size of defects.

\section{Conclusion}

The detection methods of adhesive failure in solid rocket motor include ultrasonic method, $\mathrm{X}$ ray radiography technique, industrial $\mathrm{CT}$, infrared heat wave method and laser holographic interferometry in which ultrasonic method and $\mathrm{X}$ ray radiography technique are relatively mature. Restricted by test principle and the complexity of structure of solid rocket motor, these methods cannot detect all the types of debonding.

Ultrasonic method has advantage in its low costs and high degree of automation. But conventional ultrasonic method cannot detect adhesive failure in multi-layered interface. X ray method cannot inspect the interface kissing damage. $\mathrm{X}$ ray detection must be done in radiation protection facility for its radiological hazard. And this restrains $\mathrm{X}$ ray method from solid rocket motor with heavy calibre and long size. $\mathrm{X}$ ray radiography technique has a long detection period and high cost of film. The judge of film should be taken in darkroom with film viewer. X ray real-time imaging method can imaging the specimen in real time. Compared with $\mathrm{X}$ ray radiography technique, X ray real-time imaging method has a lower detection sensitivity. Industry CT method shows the detecting result intuitively. Nevertheless, ICT method can hardly be used to detect large-scale rocket motor. And the ICT facility is expensive with low detection efficiency. The precision of ICT method is concerned with the image reconstruction method. The specimen inspected by infrared heat wave method is restricted on the thickness of shell and the heat conductivity coefficient of materials. Infrared heat wave method can only test the defect in several millimeters. Laser holographic interferometry can merely detect the adhesive failure between cladding and propellant grains.

Above all, it's better to use ultrasonic Lamb waves method and infrared heat wave method to detect adhesive failure between rocket motor shell and liner. The detecting of debonding between cladding and grains prefers to laser holographic interferometry. ICT method is suit to detecting multi-layered debonding in solid rocket motor which is not large. By means of improving signal processing algorithm and modifying image reconstructing method and developing new technology as phased array ultrasonic, the capacity of detecting debonding in rocket motor will get a further improvement.

\section{References}

[1] Tan Guangming,Bu Zhaoxian,Zhang Xiaohong. Solid Propellant Charge Design[M]. Beijing: National Defense Industry Press, 2013.

[2] Zhang Xuzhu. Double Base Propellant[M]. Beijing: Beijing Institute of Technology Press, 1997.

[3] Liu Ziru,Shao Yinghui,Ren Xiaoning,Chang hai. The Mathematical Model of Life Prediction and Its Calculation[C].The Seminar of Safety and Life Prediction of Explosives and Powders and Charges, Xi'an,2014:1-10.

[4] Xu Xuewen,Xing Yaoguo,Peng Jun. Review of Research on the Crack Risk Present in the Solid 
Rocket Motor Grain[J]. Journal of Naval Aeronautical Engineering Institute, 2007, 22(1):101-105.

[5] Zhao Yongjun,Zhang Xinggao,Zhang Wei,Zhu Hui,Wang Chunhua. Review on the Aging Property of Solid Propellant and Bonding Interface Abroad[J]. Journal of Rocket Propulsion, 2008,34(3):35-38.

[6] Jiang Shufang,Guo Xingwang,Shen Jingling,Zhang Cunlin. Infrared Thermal Wave NDT on the Disbonds of the Heat Insulated Layer in Solid Propellant Rocket Motors[J].Laser \& Infrared, 2005,35(8):584-586.

[7] Maldague,Xavier P V,Moore,et al. Nondestructive testing handbook: Infrared and thermal testing[M]. New York:ASNT,2001.

[8] Chen Guicai,Wu Dongliu,Cheng Chayuan,Guo Guangping. Non-destructive Evaluations of Composite Debonding Flaws by Infrared Thermal Wave[J]. Aerospace Materials \& Technology,2004,(1),55-58.

[9] Fan Dandan,Pan Jinxiao,Liu Bin,Liu Zilong. Research of Thermal Excitation Method in Infrared NDT for Multiple Interface Debonding[J].Laser \& Infrared, 2011,41(3):284-287.

[10]Guo Xingwang,Li Ransheng,Ding Mengmeng. Simulating Modulated Thermography of Cladding Debond in Solid Rockets[J]. Journal of Mechanical Engineering, 2011,47(2):9-15.

[11]Jin Guofeng,Zhang Wei,Yang Zhengwei,Song Yuanjia,Tian Gan. Application of Ultrasonic Infrared Thermal Wave Technique in Detection and Recognition of Interface Kissing Damage [J].Journal of Sichuan University (Engineering Science Edition), 2013, 45(2):167-175.

[12] Wu Cuiqin,Hong Xinhua,Wang Weiping,Yuan Qigang,Li Yanjun,Zhang Wei. Infrared Thermography Nondestructive Testing of Debond Defects in Composite Materials[J].High Power Laser And Particle Beams,2011,23(12):3271-3274.

[13]Bai Yang. Research on Flaw Characteristic Extraction of UT Echo Signal and Neural Network Identification for Solid Propellant of Rocket Engine[D]. Taiyuang: North University of China,2009.

[14]Green R E. Non-contact ultrasonic techniques[J]. Ultrasonics,2004,(42):9-16.

[15]Yuan Hua. Research on Ultrasonic Testing of Multi-Shell Thickness Adhesive Interface for the motors[D]. Taiyuang: North University of China,2008.

[16] Wang Zhaoba,Yang Fengbao,Chen Jun. A New Developed Ultrasonic Inspection Technology for the Cladding Quality in Solid Rocket Motors[J].Journal of Test and Measurement Technology,2001,15(3):149-153.

[17]Chen Youxing. Method and Signal Processing for Ultrasonic Testing of Multi-Layered Adhesive Interface[D].Taiyuang: North University of China,2008.

[18]Huang Pei. Study on Phased Array Ultrasonic Technology of Multi-layered Interfacial Debonding[D].Taiyuang: North University of China,2010.

[19]Zhao Peng. Study on the signal processing technology for ultrasonic testing of the liner grain debonding based on EMD[D]. Taiyuang: North University of China,2013.

[20]Ai Chun-an,Liu Yu,Cai Kun. Acousto-ultrasonic Detection for Interface Defect Between Case and Insulation of SRM[J].Journal of Solid Rocket Technology,2009,32(1):114-118.

[21]Lu Minghui,Wang Haifang,Chen Yi-Fang. Ultrasonic feature scan imaging detection system for solid rocket grain[J]. Journal of Solid Rocket Technology,2007,30(5): 459-462.

[22]Li Guohua,Wu Miao. Modern Non-destructive Testing \& Evaluation[M]. Beijing: Chemical Industry Press,2013.

[23]Zheng Wei,Deng Anhua,Liu Yunfeng,Liu Rongzhen. High Energy X-Rays Testing Technology 
of Solid Rocket Motor Debonds Between Propellant and Liner[J]. Journal of Naval Aeronautical and Astronautical University,2014, 29(4):355-359.

[24]Ai Chun-an,Sang Yuan,Xu Zhigao,Liu Yu. Solid Rocket Motor Sectional View Reconstruction form X-Ray Films[J]. Nondestructive Testing,2011,33(3):1-4.

[25]Liu Fugang,Shang Yupei,Wang Li. Study on High Energy X-Rays Testing technology of Solid Rocket Motor[J]. Nondestructive Testing,2005,29(5):10-12.

[26]Zhang Lihan,Li Hongyan,Wang Ying,Chen Xueli,Xue Qi. X-Ray Applied In Testing the Interface Between the Propellant Surface and its Coating[J]. Nondestructive Testing,2013,35 (4):60-64.

[27]Luo Jinghua,Duan Rusheng. Nondestructive Testing of Interface Bond Quality of Propellant and Cladding[J].Aero Weaponry,1990,(5):20-22.

[28]Wang Dongsheng,Ma Zhaoguang,Wang Weiming. Testing of detect for solid grain coating by laser holographic interferometry[J]. Infrared and Laser Engineering,2013, 42 (2) :376-380.

[29] Wu Wenjun, Qu Jun,Zhou Hao. Laser Shearography Testing for Interface Debond Between Grain and Liner[J]. Nondestructive Testing,2010,32(5): 373-375.

[30]Li Huijuan,Zhang Jingtao,Huang Zhenhua. Quantitative Measurement for Disbanding Defect Using Shearography[J].Aerospace Materials \& Technology,2011,(5):85-88.

[31]Zi Peng. Research on the Quanitiative Assessment System about the Claddings'Debonding Defects by Laser Holographic Test[D].Nanchang: Nan Chang Hang Kong University,2011. 